\title{
A load shedding scheme against both short- and long-term voltage instabilities in the presence of induction motors
}

\author{
Bogdan Otomega Member, IEEE
}

Abstract-In the presence of induction motors in loads, longterm voltage instability may result in sharp voltage decreases, once the generators supporting transmission voltages have their field currents limited. Furthermore, after a fault, induction motors may fail re-accelerating and their stalling leads to shortterm voltage instability. This paper investigates the ability of the load shedding scheme, previously proposed by the authors, to deal with both short and long-term voltage instabilities. The scheme is distributed with minimal amount of information exchange. It is also shown that the selectivity of this protection scheme can be increased by prioritizing in time the various controllers. Detailed time simulations of a test system are reported.

Index Terms-Long-term voltage instability, short-term voltage instability, emergency control, undervoltage load shedding, induction motors, distributed control

\section{BACKGROUND}

Load shedding is a cost-effective countermeasure against voltage instability triggered by large disturbances [1], [2], [3]. To this purpose some event-based [4] and response-based [5], [6], [7], [8] load shedding protection schemes have been successfully developed and tested. The latter allow to adjust the corrective action to the disturbance severity and location and to operate in closed loop for higher robustness.

This paper is the continuation of Ref. [9], where the impact of the induction motor loads on a load shedding protection scheme designed to deal with long-term voltage instability, initialy proposed in [10], was under study. For the load shedding scheme to cope with the fast response of motor loads, additional information exchange was required in order to enable the protection action with a reduced time delay. Therefore, the protection scheme changed from a purely distributed to wide-area, although simplicity was maintained. Moreover, the latter preserved important features such as closed-loop operation and redundancy between controllers. The proposed scheme was successfully tested under severe situation, as it was assumed that only non-motor loads can be shed.

B. Otomega (bogdan.otomega@yahoo.com) is senior lecturer in the Power Systems Dept. of the "Politehnica" University of Bucharest, Romania.

T. Van Cutsem (t.vancutsem@ulg.ac.be) is a research director of the Fund for Scientific Research (FNRS) and adjunct professor in the Dept. of Electrical Engineering and Computer Science (Montefiore Institute) of the University of Liège, Belgium.

The work has been co-funded by the Sectoral Operational Programme Human Resources Development 2007-2013 of the Romanian Ministry of Labour, Family and Social Protection through the Financial Agreement POSDRU/89/1.5/S/62557

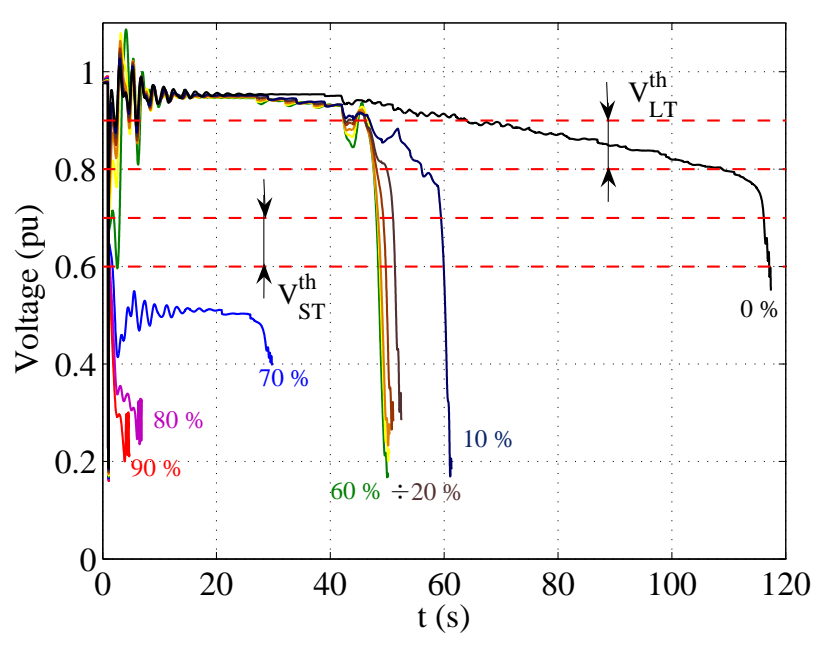

Fig. 1. Typical voltage thresholds for short- and long-term unstable cases

Induction motors due to their ability to reaccelerate after a fault, play an important role in short-term voltage instability. The objective of the paper is to report on the extension of the protection scheme dealing with short-term voltage instability.

Furthermore, this paper will also compare the effect of shedding motor vs. non-motor loads.

\section{PRINCIPLE OF THE COMBINED PROTECTION SCHEME}

\section{A. Protection against long-term voltage instability}

The most important settings of an undervoltage load shedding scheme are the voltage threshold $V^{\text {th }}$ below which the controller starts curtailing load, and the delay $\tau$ before loads are effectively disconnected.

In long-term voltage instability scenarios, where voltage degradation is precipitated by generator field current limitations, the voltage threshold value $V_{L T}^{t h}$ has to be set high enough, typically in the range $\left[\begin{array}{ll}0.8 & 0.9\end{array}\right] \mathrm{pu}$, as illustrated in Fig. 1 with the rightmost curve. The main consequence is that the corresponding delay $\tau_{L T}$ should be large enough in order not to shed load inadvertently in case of a normally cleared fault. This is illustrated in Fig. 2, showing the minimum delay $\tau_{L T}^{\min }$ that could be chosen when $V_{L T}^{t h}$ is set to $0.90 \mathrm{pu}$. Shorter delays could be considered if, at the same time, $V_{L T}^{t h}$ was set to a lower value.

In the presence of induction motor loads, the final voltage collapse can be very fast, as illustrated by the five curves in the middle of Fig. 1, corresponding to various proportions 


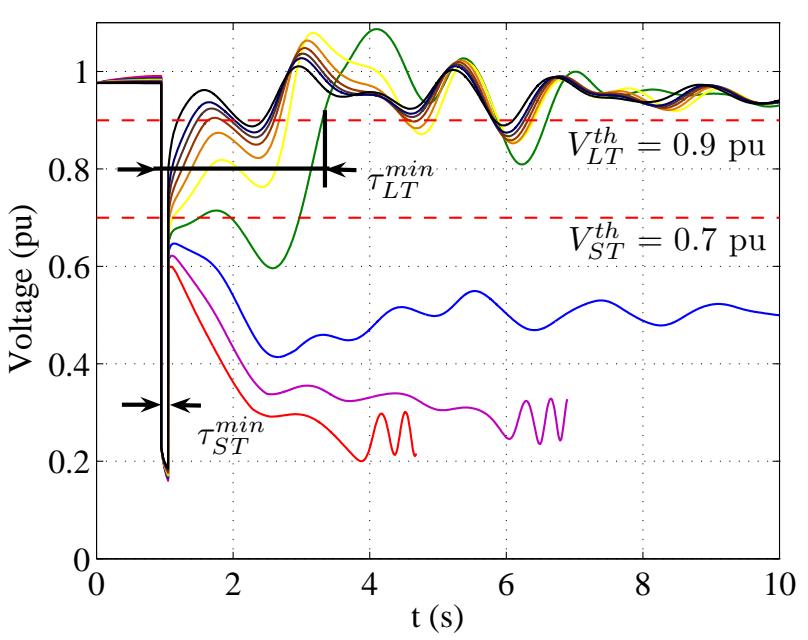

Fig. 2. Minimum shedding delays for short- and long-term unstable cases

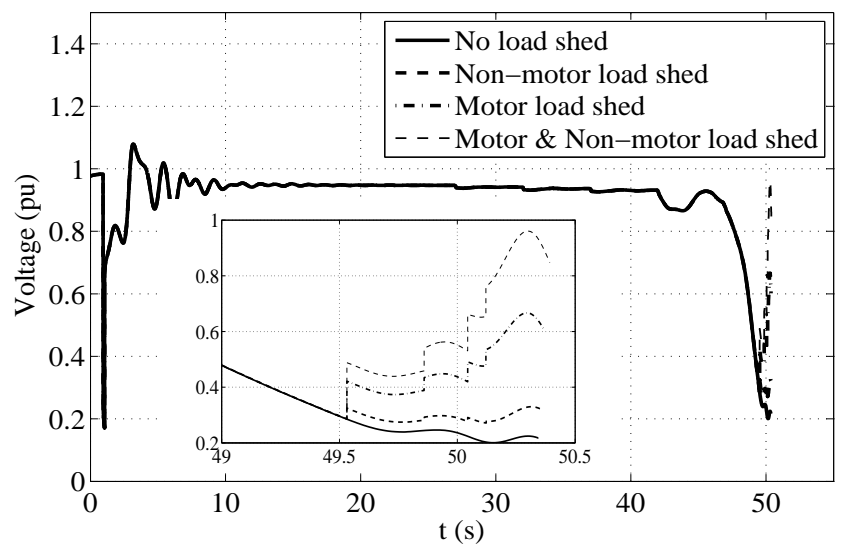

Fig. 3. Voltage evolution for different shedding combinations for $50 \%$ of motor load

of motor load (for the same system, disturbance and initial load power). If the above mentioned delay $\tau_{L T}$ is used, the controllers are too slow and fail stabilizing the system. This is illustrated in Fig. 3, in which the controllers (with $\tau_{L T}$ set to $3 \mathrm{~s}$ ) cannot prevent the voltage from reaching very low values, no matter whether the motor part, the exponential load part or both parts of loads are curtailed. To face this sharp voltage drop, the activation delay of the load shedding controllers should be decreased. However, as already mentioned, in order to ensure security of the system protection scheme, the time delay must be kept above a certain value.

In order to reconcile these two important requirements, an extension bearing the spirit of wide-area protection [5] was developed and reported in [9]. The idea is to send to the controllers an additional signal allowing them to significantly decrease their activation delay $\tau_{L T}$. To this purpose, since the voltage fall is precipitated by the activation of generator OverExcitation Limiters (OEL), it is appropriate to use the overexcitation information from generators temporarily exceeding their field current limits.

A graphical representation of the load shedding scheme logic proposed in [9] is given in Fig. 4. Since a generator

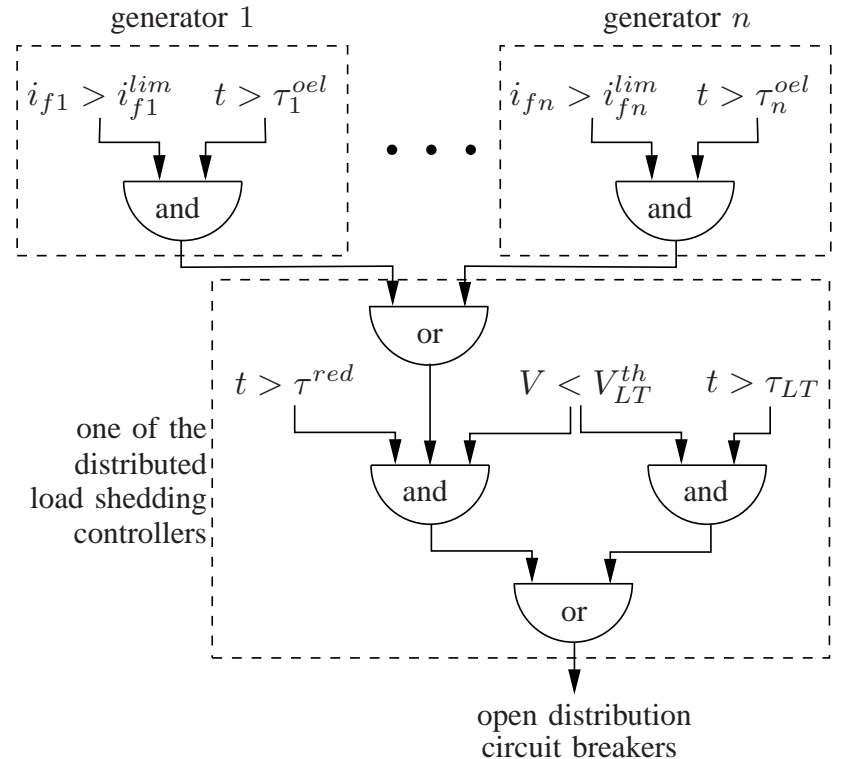

Fig. 4. Logic of the wide-area protection scheme to counteract long-term voltage instability

field current may temporarily exceed its OEL limit, under the effect of a nearby fault, the current $i_{f i}$ of the $i$-th generator has to remain above the limit for some time $\tau_{i}^{\text {oel }}$ before sending the signal, so that the latter truly reveals a situation where the generator is overexcited owing to its voltage control. When the OEL signal from at least one of the generators in the vicinity of the load shedding controller is received, the shedding delay is set to the lower value $\tau^{r e d}$. Of course, it is still required to have $V<V_{L T}^{t h}$ in order load shedding to be triggered. If a fault occurs near the load shedding controller, its voltage $V$ will temporarily fall below $V_{L T}^{t h}$ but since no signal is received from the generator OELs, the controller will not react before $\tau_{L T}$ seconds. Assuming that the fault is cleared normally and voltage recovers above $V_{L T}^{t h}$ in less than this time, the controller is merely reset.

\section{B. Protection against short-term voltage instability}

Short-term voltage instability is mainly associated with the stalling of induction motors after a fault [1], [11]. If they do not stall but do not re-accelerate fast enough, a pronounced voltage dip may also result, which can be unacceptable for sensitive loads. This could also justify resorting to load shedding [12]. The voltage decay is obviously much faster than in case of long-term instability, as illustrated by the leftmost curves in Fig. 1. The corresponding curves in Fig. 2 show voltages "locked" at very low values after fault clearing.

Hence, the corresponding shedding delay $\tau_{S T}$ should be as short as possible, although large enough to avoid reacting to a normal fault clearing followed by a stable recovery of voltages. Luckily, the voltages are quite depressed and hence, the voltage threshold can be set to a value $V_{S T}^{t h}$ significantly lower than $V_{L T}^{t h}$ (see Fig. 1).

It is thus proposed to extend the long-term control logic of Fig. 4 by adding the short-term counterpart, which leads to the logic detailed in Fig. 5. With this extension, the proposed 


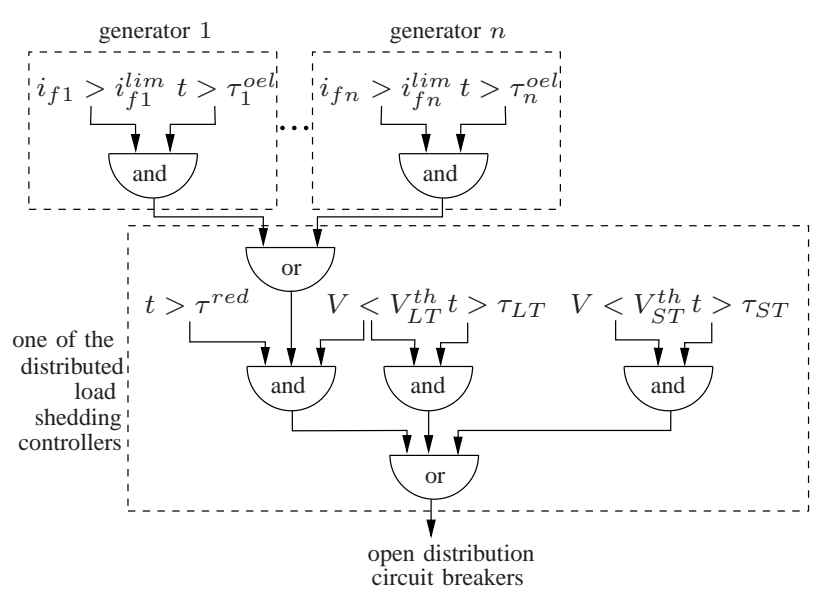

Fig. 5. Logic of the wide-area protection scheme to counteract both shortand long-term voltage instability

wide-area protection scheme is expected to properly deal with both short- and long-term voltage instability situations.

\section{Simulation RESUlts}

\section{A. Test system}

The extended load shedding scheme has been tested on the Nordic32 test system, as in [9]. Its one-line diagram is shown in Fig. 6. The model includes 55 buses, 20 generators, and 22 loads. The long-term dynamics are driven by Load Tap Changers (LTC) and OELs acting with various delays. The generators are assumed to be provided with an excitation system that takes the least demanding among the Automatic Voltage Regulator (AVR) and OEL signals. Hence, when load shedding brings relief to the generator, the OEL resets and the AVR regains control with negligible delay. All loads are connected to MV distribution buses controlled by LTCs.

The load shedding controllers are installed in the "Central" area where the largest voltage drops are observed. Each controller monitors the voltage of one transmission bus and controls the load on the distribution side of the transformer connected to that bus, as detailed in Table I. The tests reported in this paper considered a proportion of $50 \%$ of motor load for the cases leading to long-term voltage stability, and $80 \%$ of motor load for the short-term voltage instability cases.

TABLE I

CONSIDERED CONTROLLERS AND LOAD

\begin{tabular}{|c|c|c|c|}
\hline $\begin{array}{c}\text { Controller } \\
\text { name }\end{array}$ & $\begin{array}{c}\text { Monitored } \\
\text { bus }\end{array}$ & $\begin{array}{c}\text { Controlled } \\
\text { load bus }\end{array}$ & $\begin{array}{c}\text { Load } \\
\text { [MW] }\end{array}$ \\
\hline$C_{1041}$ & 1041 & 9041 & 600 \\
\hline$C_{1042}$ & 1042 & 9042 & 300 \\
\hline$C_{1043}$ & 1043 & 9043 & 230 \\
\hline$C_{1044}$ & 1044 & 9044 & 800 \\
\hline$C_{1045}$ & 1045 & 9045 & 700 \\
\hline
\end{tabular}

The non-motor loads are represented with an exponential model which behaves as constant current for active power and constant impedance for reactive power, while the motor loads are represented using an equivalent induction motor model. In the latter model it is considered that the electromagnetic transients are infinitely fast and thus are replaced by algebraic

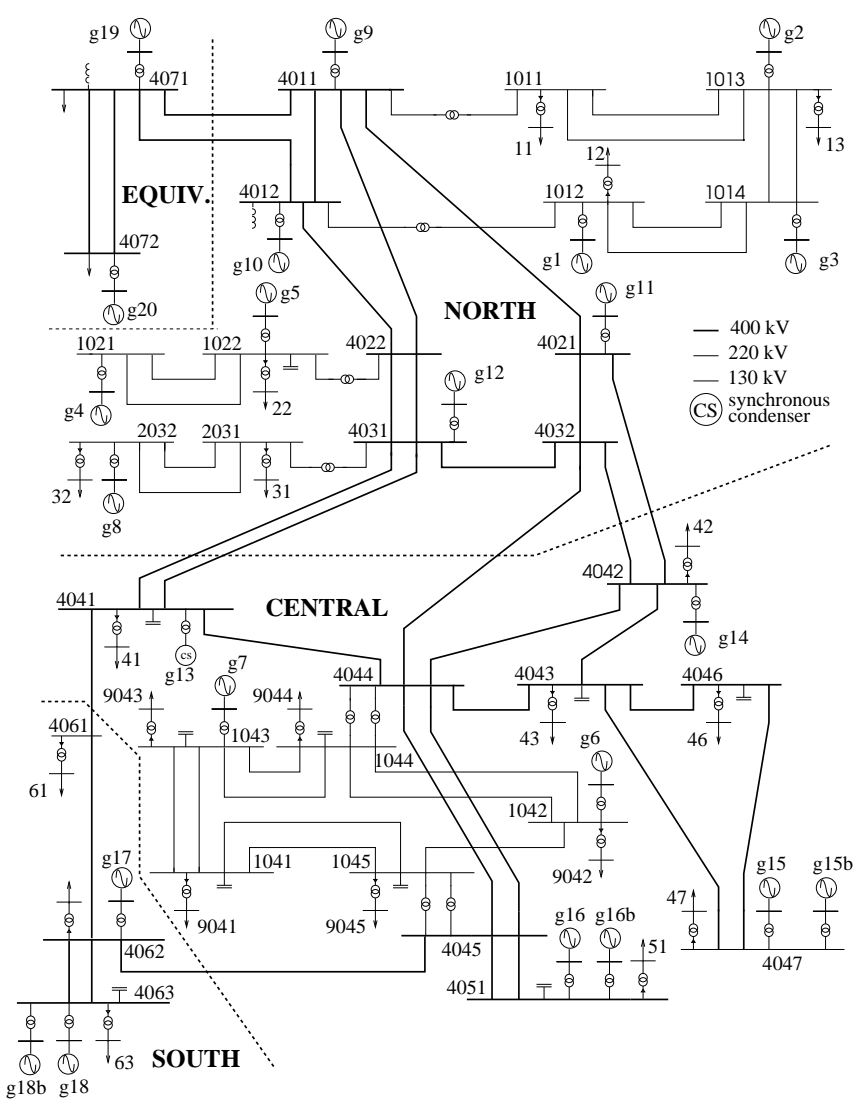

Fig. 6. Nordic32 test system

equilibrium equations, while the rotor motion dynamics are represented by a first order equation on speed. When motor load is shed the per unit motor parameters (e.g. mechanical torque) on the motor MVA base remain unchanged, but in system base they are changing as the nominal apparent power of the equivalent motor is decreasing. This change is reflected in the contributions of the motor load to the $\mathrm{Y}$ matrix.

In order to improve the power factor and voltage profile in the controlled load buses are connected shunt capacitors as illustrated in Fig. 6. Their participation is taken into account in the $\mathrm{Y}$ matrix. When shunt capacitors disconnection is considered their contribution to the $\mathrm{Y}$ matrix is changing.

The results provided in this paper relate to a set of disturbances involving the tie-lines between "North" and "Central" areas and the lines connected to bus 4044, one of the buses through which the load area under subject is supplied. In all the cases the disturbance consists of a short-circuit applied on the line, close to one of the end buses, at time instant $1 \mathrm{~s}$ and cleared after $0.1 \mathrm{~s}$ by opening the line from both ends.

\section{B. Identical controllers dealing with long-term instability}

Tests reported in [9] have shown that the proposed widearea protection scheme performs very well against long-term voltage instability. Regarding the controller settings, tests carried out with $V_{L T}^{t h}$ in the interval $\left[\begin{array}{ll}0.8 & 0.9\end{array}\right] \mathrm{pu}$ and $\tau^{\text {red }}$ taking values in the interval $\left[\begin{array}{ll}0.2 & 1\end{array}\right] \mathrm{s}$, have shown that, due to the reduced time delay values, the wide-area protection succeds to save the system for a large set $\left(\tau^{r e d}, K\right)$ combinations, no 


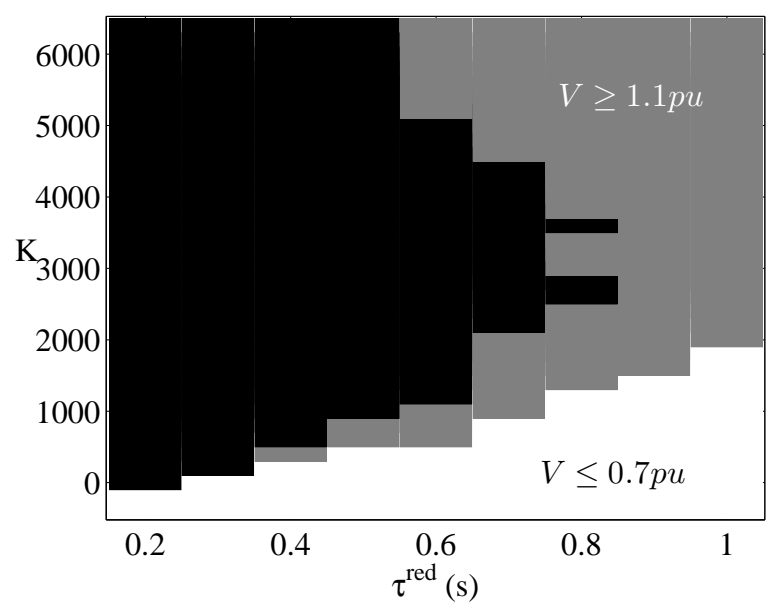

Fig. 7. Regions of successful, unsuccessful, and overvoltage operation of the load shedding scheme; non-motor load curtailed

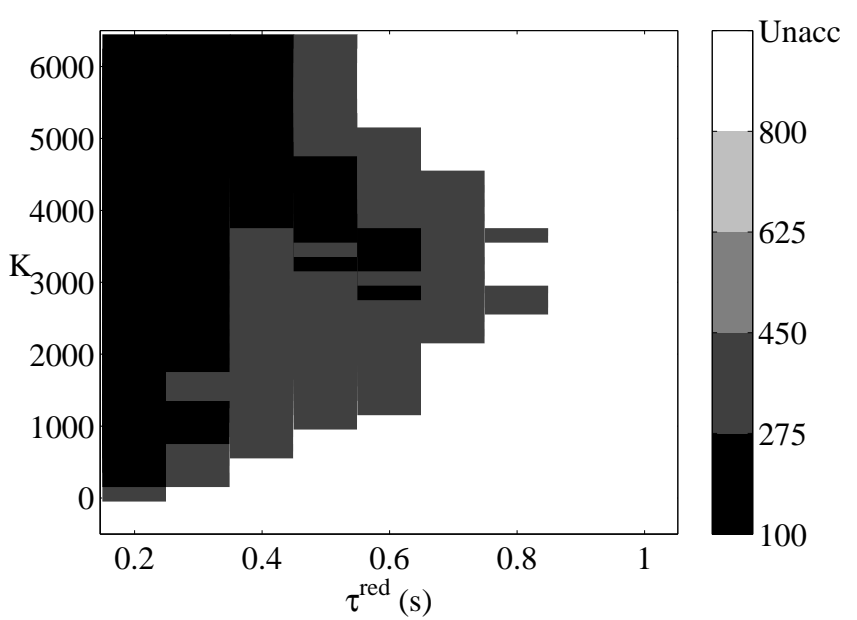

Fig. 8. Performance of load shedding scheme for various settings; non-motor load curtailed

matter the voltage threshold value. Therefore the latter has been set to $0.85 \mathrm{pu}$.

Figure 7 shows the operation regions of the protection scheme in the $\left(\tau^{\text {red }}, K\right)$ space, where $K$ relates the amount of load shedding to the average voltage drop (used as a measure of severity) [10].

$$
\Delta P^{s h}=K \cdot \frac{1}{\tau^{r e d}} \int_{t_{0}}^{t_{0}+\tau^{r e d}}\left(V^{t h}-V(t)\right) d t
$$

The white region corresponds to failure to stabilize the system, with $V$ falling below $0.7 \mathrm{pu}$. The grey region corresponds to $\left(\tau^{r e d}, K\right)$ combinations for which the system is stabilized but experiences overvoltages, with some transmission voltages settling at values above $1.1 \mathrm{pu}$. Finally, the black region corresponds to proper operation yielding voltages within the prescribed limits. The grey region, although not desirable from operation viewpoint, is of course preferable to the white one.

From the large set of $\left(\tau^{r e d}, K\right)$ combinations for which the protection scheme succeeds to save the system, the best one is chosen based on the amount of load shedding. To this purpose, Fig. 8 shows the total load power shed for various $\left(\tau^{r e d}, K\right)$ combinations inside the black region in Fig. 7. The

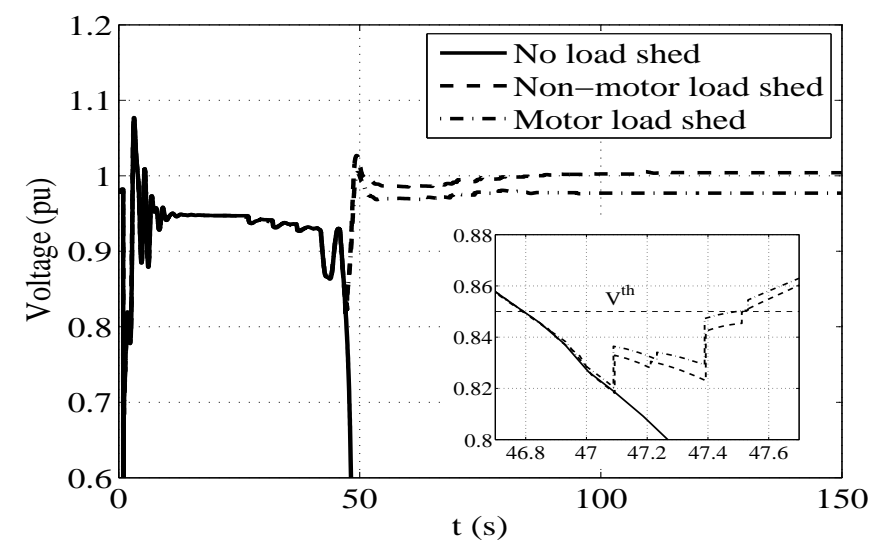

Fig. 9. Evolution of voltage at bus 1041 without and with shedding

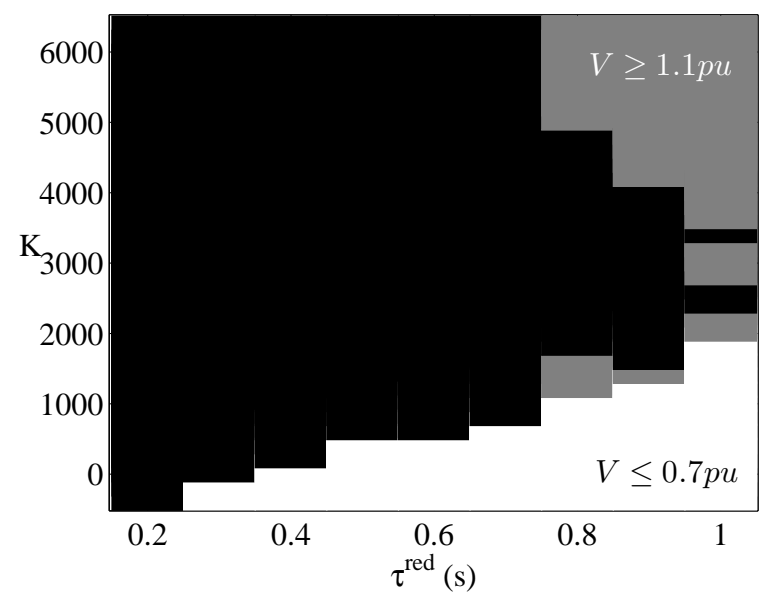

Fig. 10. Regions of successful, unsuccessful, and overvoltage operation of the load shedding scheme; motor load curtailed

best combination over the considered set of disturbances was found to be $\tau^{\text {red }}=0.3 \mathrm{~s}, K=3000 \mathrm{MW} / \mathrm{pu}$. The dashed curve in Fig. 9 shows the very satisfactory voltage evolution obtained with this setting.

In the results presented so far (and in [9]) only nonmotor loads were curtailed. In principle, this leads to a more severe situation since the whole induction motor load remains connected to the system. We present hereafter results obtained when curtailing the motor part of loads (assuming that one can access those loads inside the aggregate).

As in the case of non-motor load shedding, the rather low value of $\tau^{r e d}$ makes the protection scheme less sensitive to the choice of $V_{L T}^{t h}$, and we keep on setting this threshold to $0.85 \mathrm{pu}$.

Shedding motor loads only yields an even more reliable protection scheme. This can be seen from Fig. 10 where the region of $\left(\tau^{r e d}, K\right)$ values for which the system is stabilized, without experiencing overvoltages, is larger than its counterpart in Fig. 7 (non-motor load shedding).

Figure 11 shows the amount of (motor load) power shed. It is to be compared with Fig. 8 relative to non-motor load shedding. For a majority of $\left(\tau^{r e d}, K\right)$ combinations for which the system is stabilized in both cases, less load is disconnected when induction motor load is shed, for instance 193 MW vs 


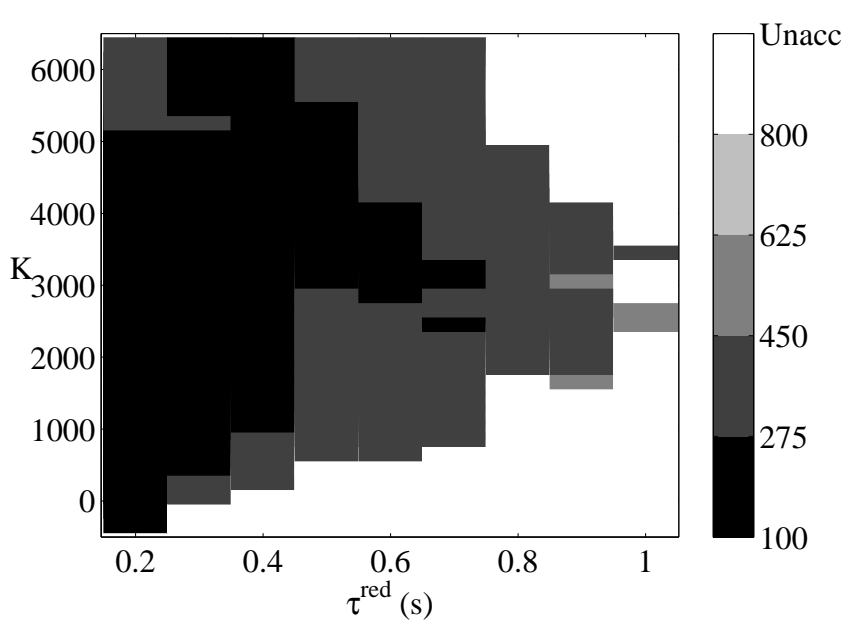

Fig. 11. Performance of load shedding scheme for various settings; motor load curtailed

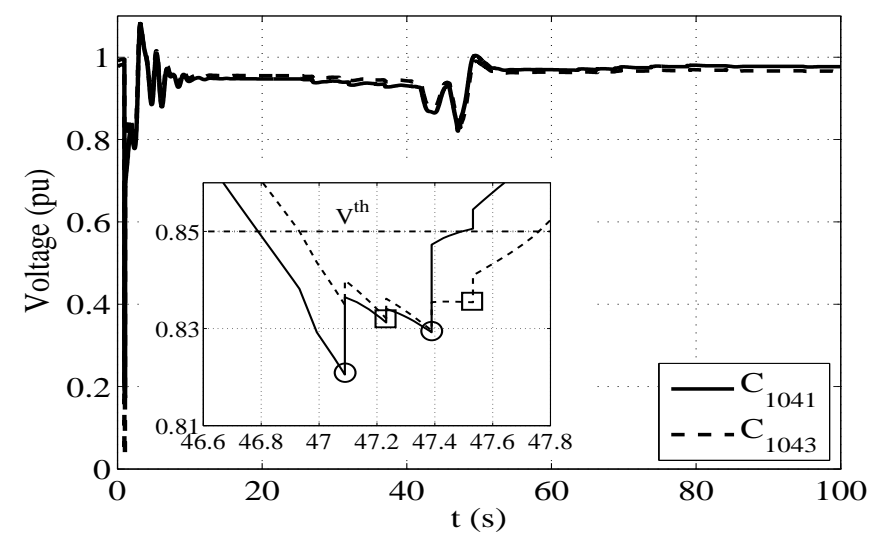

Fig. 12. Voltage evolution and controller load shedding actions

$238 \mathrm{MW}$ for the previously chosen combination of $\tau^{\text {red }}$ and $K$ values. This is explained by the fact that the voltage is more sensitive to induction motor load shed, as can be seen from (the zoomed part) in Fig. 9, where the first amount of power shed (51 MW) is identical for both shedding types. The same figure shows that the scheme acts in almost the same time.

Finally, Fig. 12 shows the evolution of the bus voltages monitored by the two controllers responding to the voltage sags. In the same figure, the circles and squares indicate the load shedding actions of controllers $C_{1041}$ and $C_{1043}$, respectively. It can be seen that the whole load shedding takes place in a little more than 0.5 seconds. This suggests that some more coordination between the controllers might yield lower load curtailments. We recall indeed that the load shedding scheme used in this work is purely distributed, i.e. the controllers interact only through the transmission voltages that they monitor [10]. An improvement preserving the simplicity of this distributed scheme is discussed and illustrated in the next subsection.

\section{Time-prioritized controllers dealing with long-term insta- bility}

One way of increasing the coordination between controllers would consist in adding information exchange between them.

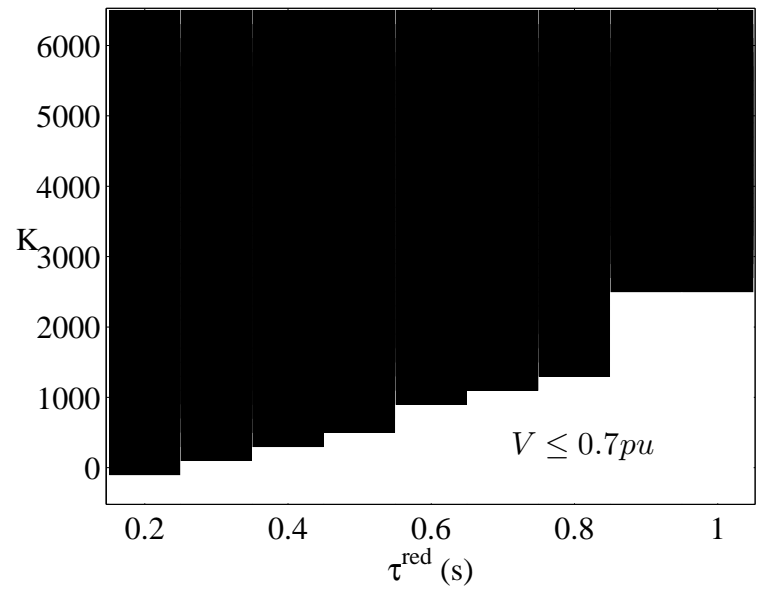

Fig. 13. Regions of successful and unsuccessful operation of the load shedding scheme; motor load shedding and time-prioritized controllers

For instance, one could think of allowing only the controller with the largest voltage drop to act at a time and resetting all controller time counters when one of them acts. However, this information exchange would make the protection scheme more complicate and, hence, less reliable. The impact of communication delays should be also carefully assessed.

A much easier (implicit) coordination consists in making the controllers react with various delays, so that there are more chances that a controller with lower priority in time does not act, or acts less, owing to the voltage increase taking place over the longer monitoring time interval. Furthermore, the initial voltage drop observed in simulations is used to prioritize the controllers. A smaller delay is assigned to a controller experiencing a larger voltage drop once the voltage instability process initiates.

Table II shows the controller ranking considered in the tests (and valid for the considered set of disturbances), together with the associated shedding delays. We have to mention that a generalised ranking has to take into account, beside a set of disturbances, different operating conditions and system topologies.

TABLE II

SHEDDING DELAYS OF CONTROLLERS RANKED ACCORDING TO VOLTAGE DROPS

\begin{tabular}{|c|c|c|}
\hline Rank & Controller & Delay (s) \\
\hline 1 & $C_{1041}$ & $\tau^{\text {red }}$ \\
2 & $C_{1043}$ & $\tau^{\text {red }}+0.1$ \\
3 & $C_{1044}$ & $\tau^{\text {red }}+0.2$ \\
4 & $C_{1045}$ & $\tau^{\text {red }}+0.3$ \\
5 & $C_{1042}$ & $\tau^{\text {red }}+0.4$ \\
\hline
\end{tabular}

The benefit of those delays is twofold. Firstly, the system experiences significantly less overvoltages. This yields a protection scheme with a significantly larger feasible region in the $\left(\tau^{r e d}, K\right)$ space, as shown by Fig. 13. Secondly, the amount of load shedding decreases for a majority of $\left(\tau^{\text {red }}, K\right)$ combinations, as can be seen from Fig. 14. 


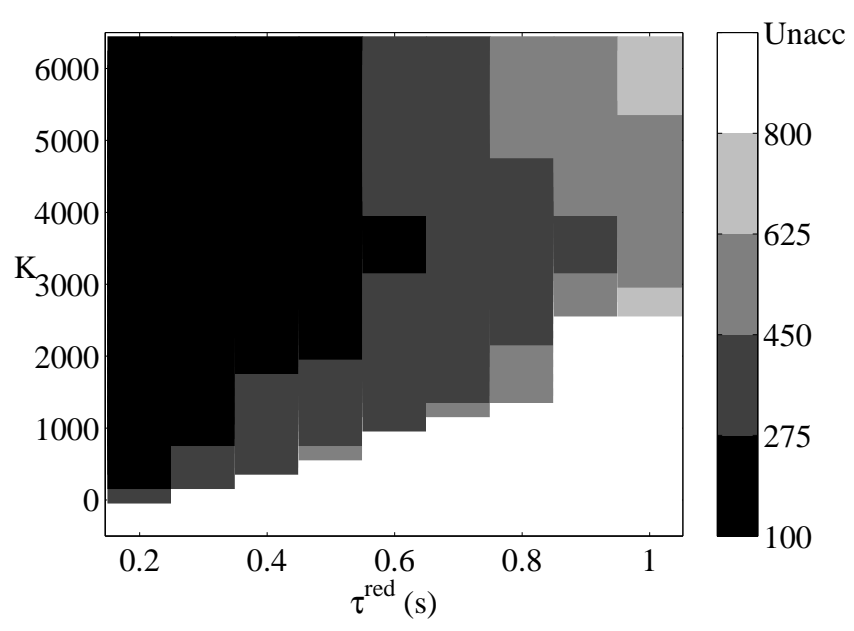

Fig. 14. Performance of load shedding scheme for various controller settings; motor load shedding and time-prioritized controllers

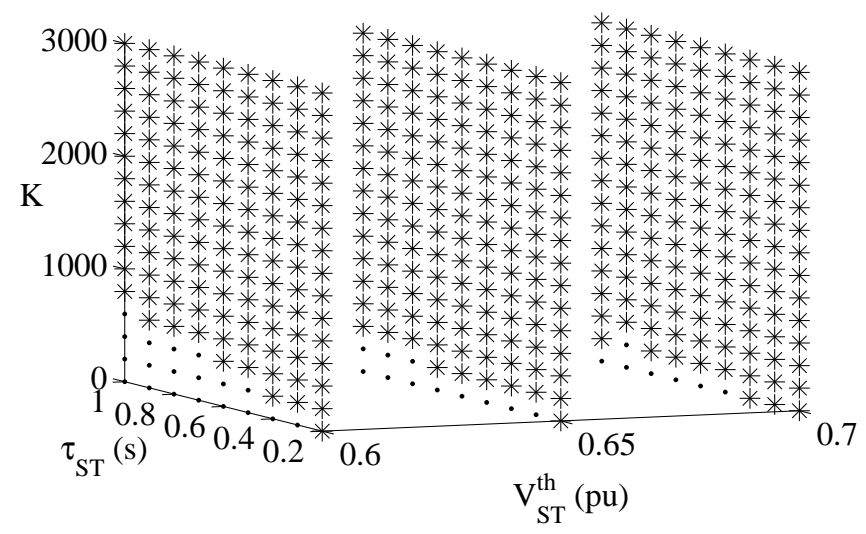

Fig. 15. Performance of load shedding scheme for various controller settings; motor load curtailed

\section{Identical controllers dealing with short-term instability}

The remaining of the paper focuses on the performance of the protection scheme to counteract short-term voltage instability. We assumed a proportion of $80 \%$ of induction motors in the loads of buses 1041 to 1045 , which leads to the fast instability shown in Fig. 1.

The overall performance of the shedding scheme is shown in Fig. 15 for various values of $V_{S T}^{t h}, \tau_{S T}$ and $K$, respectively. All controllers have been assigned the same settings. A star in the figure indicates a combination for which the system has an acceptable post-disturbance evolution, while a dot indicates a failure to save the system. The system evolution is considered acceptable if, after load shedding, no voltage falls below $0.70 \mathrm{pu}$.

As already observed for long-term voltage instability, the short shedding delays make the load shedding scheme less sensitive to the voltage threshold $V_{S T}^{t h}$, as can be seen in Fig. 15. Therefore, the latter has been set to $0.7 \mathrm{pu}$, which makes the protection scheme react faster. The results reported hereafter have been obtained for that setting.

Next, the best combination of $\left(\tau_{S T}, K\right)$ values was determined from performance diagrams of the type shown in Fig. 16, the short-term counterpart of Fig. 8. The best combi-

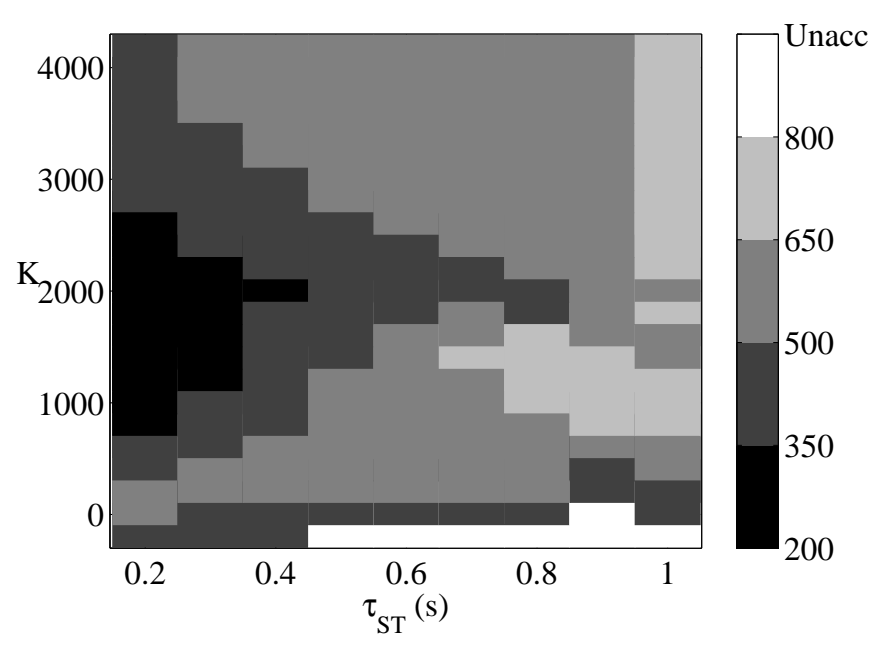

Fig. 16. Performance of short-term load shedding scheme for various settings; $V_{S T}^{t h}=0.7 \mathrm{pu}$

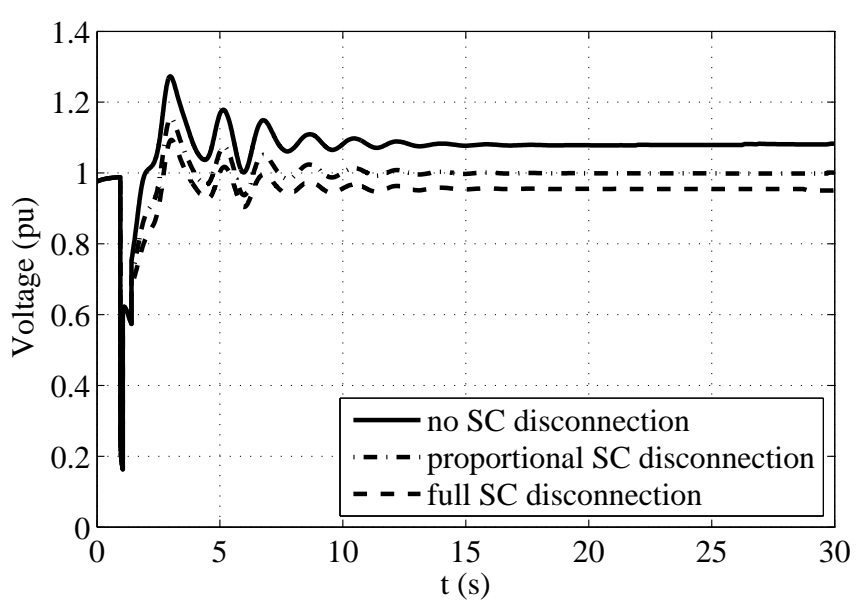

Fig. 17. Evolution of voltage at bus 1041 for various strategies of shunt capacitor disconnection

nation over the set of disturbances under analysis was found to be $\tau_{S T}=0.3 \mathrm{~s}, K=2600 \mathrm{MW} / \mathrm{pu}$.

Initial tests revealed that the system experiences overvoltages shortly after the load shedding actions. An example of voltage evolution is provided in Fig. 17, while Fig. 18 shows the corresponding load power curtailments by the two responding controllers. The solid curve in Fig. 17 shows a clearly unacceptable voltage increase. This results, on one hand, from the important amount of load shed in a very short time interval and, on the other hand, from the important amount of shunt capacitors (aimed at improving load power factor) that remains connected at four out of five load buses controlled by the load shedding controllers.

In order to avoid such overvoltages, two strategies regarding shunt capacitor disconnection have been considered. The first strategy consists of disconnecting shunt compensation in the same proportion as motor load. In the second strategy, it was considered that if more than $50 \%$ of the load active power is shed, then the whole shunt compensation is disconnected. The resulting voltage evolutions are shown with dashed-dotted and dashed lines, respectively, in Fig. 17. Clearly, the overvoltage 


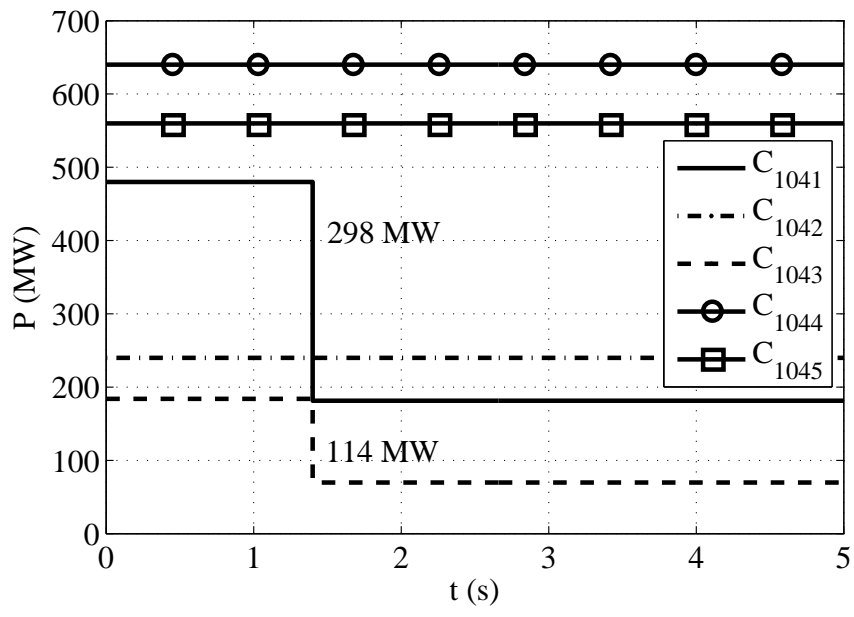

Fig. 18. Time evolution of the active power shed; same scenario as in Fig. 17

problem is mitigated. The results of the second strategy are better and, although it might be a bit more difficult to implement, it was used when producing the results shown in Figs. 15 and 16.

\section{E. Time-prioritized controllers dealing with short-term insta- bility}

Figure 18 shows a typical situation where the shedding condition is satisfied for two controllers at the same time, which leads them to act simultaneously. This is the price to pay for the lack of information exchange between controllers. However, some improvement can be expected by prioritizing the controllers, as was done for long-term instability (see Section III-C).

The shedding delays $\tau_{S T}$ have thus been adjusted. The values in Table II have been re-used, since $\tau^{r e d}=\tau_{S T}=0.3 \mathrm{~s}$.

Figure 11 shows the total motor load power shed by the so adjusted protection scheme. It can be seen that the additional delays do not influence the overall protection scheme reliability (in terms of $\left(\tau^{r e d}, K\right)$ combinations for which the system is stabilized). On the other hand, as expected and already observed with long-term instability, less load is shed. For example, in the case presented in Fig. 18, the sole action of controller $C_{1041}(298 \mathrm{MW})$ is enough to restore all the monitored voltages above the voltage threshold and stabilize the system.

\section{CONCLUSION}

A load shedding scheme has been presented, able to deal with both long- and short-term voltage instabilities in the presence of induction motors in loads. The scheme is distributed and the controllers do not exchange information, which yields higher simplicity and hence reliability. The only communication (although not critical in terms of adding response delays) is a signal sent to the controllers indicating that neighbouring generators are going to have their field current limited, and enabling them to shed load faster. This signal is used when counteracting long-term voltage stability.

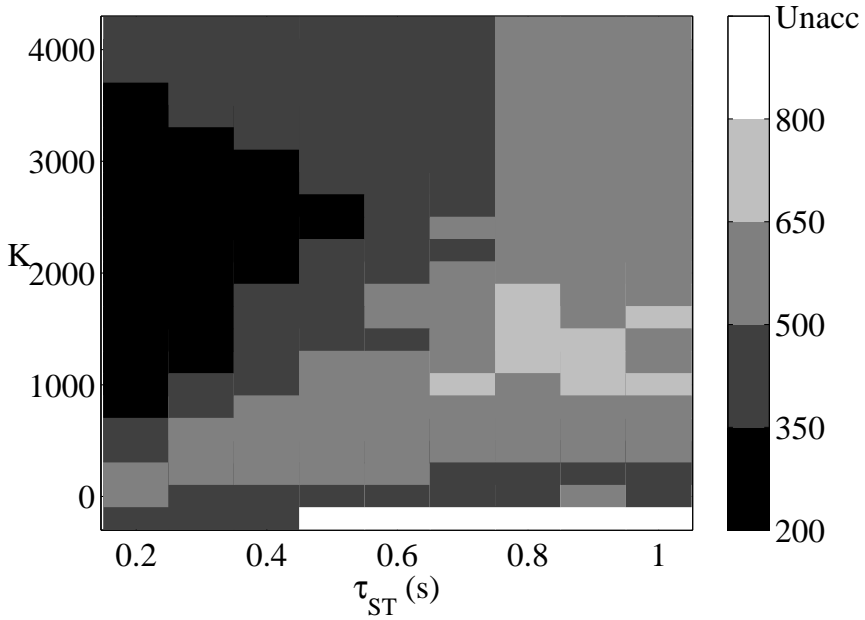

Fig. 19. Performance of short-term load shedding scheme for various settings; time-prioritized controllers

The scheme has been complemented with its short-term counterpart, involving lower voltage thresholds and directly fast response.

In short- and long-term instability cases, an improved coordination between the fast responding controllers can be obtained by time prioritizing them. This leads to curtailing lower amounts of load, increases the range of satisfactory operation and reduces the risk of overvoltages, while preserving the distributed feature of the scheme.

Additional results have been given and discussed regarding the effect of shedding motor vs. non-motor load and emphasizing the need to disconnect the compensation shunt capacitors together with motors.

\section{REFERENCES}

[1] C. W. Taylor,"Concepts of undervoltage load shedding for voltage stability", IEEE Trans. on Power Delivery, Vol. 7, 1992, pp. 480-488.

[2] S. Arnborg, G. Andersson, D.J. Hill, I.A. Hiskens, "On undervoltage load shedding in power system," Int. Journal of Electrical Power and Energy Systems, vol. 19, 1997, pp. 141-149.

[3] D. H. Karlsson (convener), System Protection Schemes in Power Networks, Final report of CIGRE Task Force 38.02.19, June 2001.

[4] V. C. Nikolaidis, C. D. Vournas, "Design Strategies for Load-Shedding Schemes against Voltage Collapse in the Hellenic System", IEEE Trans. Power Syst., Vol. 23, No. 2, May 2008.

[5] B. Ingelsson, P. Lindström, D. Karlsson, G. Runvik, J. Sjödin, "Special Protection Scheme against Voltage Collapse in the South Part of the Swedish Grid," Proc. CIGRE Conference, 1996, Paper No 38-103

[6] D. Lefebvre, C. Moors, T. Van Cutsem, "Design of an undervoltage load shedding scheme for the Hydro-Qu'ebec system", Proc. IEEE PES General Meeting, Toronto (Canada), July 2003.

[7] S. Imai, "Undervoltage load shedding improving security as reasonable measure for extreme contingencies", Proc. IEEE PES General Meeting, San Francisco (USA), 2005.

[8] F. Capitanescu, B. Otomega, H. Lefebvre, V. Sermanson, T. Van Cutsem "Prospects of an improved system protection scheme against voltage instability in the RTE system", Proc. 16th Power System Computation Conference (PSCC), Glasgow (UK), June 2008.

[9] B. Otomega, T. Van Cutsem, "Local vs. wide-area undervoltage load shedding in the presence of induction motor loads", 2009 IEEE PowerTech Conference, June 28 - July 2, Bucharest, Romania.

[10] B. Otomega, T. Van Cutsem, "Undervoltage load shedding using distributed controllers," IEEE Trans. Power Syst., vol. 22, no. 4, pp. 18981907, Nov. 2007.

[11] T. Van Cutsem, C. Vournas, Voltage Stability of Electric Power Systems, Boston, Kluwer Academic Publishers (now Springer), 1998. 
[12] S. M. Halpin, K. A. Harley, R. A. Jones, L. Y. Taylor, "Slope-Permissive Under-Voltage Load Shed Relay for Delayed Voltage Recovery Mitigation," IEEE Trans. Power Syst., Vol. 23, No. 3, pp. 1211-1216, Aug. 2008 